\title{
Opportunities and Challenges for Prevention and Intervention in Emerging Adulthood: Introduction to the Special Issue
}

\author{
Michael J. Cleveland ${ }^{1}$ (D) Abby L. Goldstein ${ }^{2}$
}

Published online: 23 January 2019

(C) Society for Prevention Research 2019

The impetus for this special issue began as a dialogue among scientists belonging to two professional organizations - the Society for the Study of Emerging Adulthood (SSEA) and the Society for Prevention Research (SPR). At the 2015 biennial meeting of SSEA, Dr. Seth Schwartz, then-president of SSEA and Dr. Richard Catalano, then-president of SPR, organized a Presidential panel discussion on prevention science that featured three leading prevention scientists whose research focuses on emerging adults (Drs. Rob Turrisi, Robert Saltz, and Margaret Kuklinksi). The panel stimulated lively discussion among the presenters and the SSEA audience and inspired the co-editors of this special issue to organize a similar roundtable at the subsequent SPR meeting in the summer of 2016.

The SPR roundtable featured three leading scholars of emerging adult research (Drs. Abby Goldstein, MarieHélène Véronneau, and Christine Lee) and focused on exploring four questions that capture the challenges for prevention scientists seeking to address the needs of emerging adults: (1) How can existing preventive interventions be adapted to better suit emerging adults? (2) Are there alternative strategies that can reach all emerging adults? (3) What is the best timing for delivery of preventive interventions aimed at emerging adults? and (4) How can existing theories and approaches to prevention be adapted to better suit the developmental needs of emerging adults? Although it was clear from the discussion that many researchers and practitioners were implementing

Michael J. Cleveland

michael.cleveland@wsu.edu

Abby L. Goldstein

abbyl.goldstein@utoronto.ca

1 Department of Human Development, Washington State University, Pullman, WA, USA

2 Department of Applied Psychology and Human Development, Ontario Institute for Studies in Education, University of Toronto, Toronto, ON, Canada new approaches to best meet the needs of this population, the discussants and audience members agreed that there were still many gaps in the literature regarding emerging adult prevention and intervention. Because so much of the prevention work with emerging adults has focused on interventions for college student drinking, key gaps include a need for additional research on prevention programs with non-collegeattending emerging adults, as well as a greater focus on risk behaviors and mental health concerns other than alcohol use and inclusion of more diverse samples of emerging adults.

This special issue represents the next step in this scientific conversation. Our goal is to highlight current theoretical and empirical research addressing the unique prevention and intervention needs of emerging adults, with the hope that this special issue inspires scientists across disciplines to turn their attention to this developmental stage.

\section{Prevention Research and Emerging Adulthood}

During emerging adulthood, generally defined as the ages between 18 and 29 years, most young people face an array of new experiences and must navigate many changes associated with the transition into adult roles, such as establishing financial and residential independence, entering stable romantic partnerships, and securing full-time employment (Arnett 2000, 2007). Because this is a time of significant transitions, emerging adulthood is a critical period in the lifespan when possibilities for increased life satisfaction co-exist with the onset and escalation of serious psychopathology, such as major depression, anxiety, schizophrenia (Schulenberg et al. 2004), and substance use (Johnston 2010).

Despite this risk (and opportunity), the majority of preventive interventions focus on earlier stages of the lifespan, most notably childhood and adolescence. These preventive interventions frequently target delaying the initiation of high-risk behaviors. While this is an important goal when considering earlier stages of development, there is a clear need for 
approaches that address the prevention needs of emerging adults. With respect to emerging adulthood, successful interventions have been developed to target high-risk alcohol use among college students (e.g., Larimer and Cronce 2007; U.S. Department of Health and Human Services 2016), with fewer efforts targeting emerging adults in other contexts or focusing on other health-risk behaviors. Given this gap, when soliciting articles for inclusion in this special issue, we specifically requested studies that extended beyond college populations, targeted a variety of behaviors, or tested novel preventive interventions. We also emphasized the need to include not only original, empirical research examining prevention and intervention efforts developed for emerging adults but also reviews and perspectives that integrate key conceptual issues and advance theoretical knowledge.

The articles included in this special issue reflect that diversity. Following this introduction, Schwartz and Petrova (2019) provide an overview of emerging adulthood and describe specific mechanisms and conditions that are associated with health behaviors in emerging adulthood. Four of the studies evaluate the efficacy of preventive interventions that were designed to reduce common risk behaviors during emerging adulthood. Three of these evaluations describe the results of randomized controlled trials (RCTs).

Lewis et al. (2018) evaluated the efficacy of two web-based personalized feedback interventions that either integrated or simply combined alcohol and risky sexual behavior content among a national sample of 402 sexually active emerging adults (ages 18-25 years) who reported recent alcohol use. The results indicated that at the 1-month follow-up, the integrated and combined conditions were each associated with short-term reductions of alcohol-related risky sex behaviors, compared to the control condition. Notable differences in the effects of the specific intervention conditions were found, however. Whereas the combined condition showed a reduction in typical number of drinks per week, the integrated condition was associated with a reduction in alcohol-related consequences (Lewis et al. 2018).

Also using an RCT design, Stormshak et al. (2018) evaluated the efficacy of a multi-modal family-based intervention, delivered during the emerging adulthood years, in reducing risk behaviors among a community sample originally recruited during middle school. The adapted intervention was designed to target the unique features of emerging adulthood, including enhancing the parent-youth relationship and communication, encouraging healthy relationships, and promoting emerging adults' autonomy. About one third of the original intervention group participants who were randomly assigned to the middle school intervention (134 out of 386) participated in the adapted intervention, which was delivered one year after high school completion. Stormshak et al. found that random assignment alone or simple engagement in the intervention was not associated with changes in a combined index of risk behavior outcomes (marijuana use, binge drinking, risky sexual behavior, and vocational risk). However, dose-response analyses suggested that participants who were more engaged in the intervention reported reductions in risk behavior at the second emerging adult assessment, when participants were on average 21.5 years old.

Hafner and Rushing (this issue) describe an RCT trial evaluating the efficacy of Native Voices, a culturally adapted sexual health intervention for reducing sexual risk behaviors among American Indian and Alaska Native (AI/AN) youth. The single-session, group-delivered intervention was designed for delivery in STI clinics and included a video, group skill-building session, and psychoeducation. Participants were 199 AI/AN emerging adults (ages 18-24 years old) who were randomly assigned to one of three conditions: (1) sexual health fact sheets; (2) sexual health fact sheets + Native Voices video; and (3) sexual health fact sheets + Native Voices video and facilitated discussion. The primary outcome was STI risk perceptions assessed at baseline, post-intervention, and 6-month follow-up. The intervention proved ineffective at changing STI and HIV risk perceptions after accounting for baseline demographic and sexual risk factors. Hafner and Rushing (this issue) provide several possible reasons for the lack of findings, including that the intervention, which was adapted from an adult-focused intervention, may have been better suited for adolescents and not fully addressed the developmental needs of AI/AN emerging adults.

Using daily diary data and a longitudinal, measurement burst design, Layland et al. (2018) describe the effects of participation in a campus-led alcohol-free program on college students' alcohol and other substance use. Participants in the study were 730 full-time, first year students who completed 14 consecutive daily web-based surveys in each of the seven subsequent semesters of their college career. Within-person effects showed that students reported less alcohol and other substance use on weekend nights they attended the universitysponsored programming, compared to weekend nights they did not attend, across the study period. These effects were strongest among female students and those under the legal drinking age of 21.

The present special issue also includes two studies using longitudinal data to examine associations between risk factors and health outcomes among community samples of emerging adults. Using a nationally representative sample of US adults, Fish and colleagues (this issue) first compared rates of suicidal behavior between heterosexual and sexual minority (i.e., lesbian/gay and bisexual (LGB)) emerging adults across the lifespan and then examined how the association between antiLGB discrimination and suicidal behavior varied with age. Differences between sexual minority and heterosexual adults in the likelihood of recent suicidal behavior were most apparent at age 18 (20\% vs. 5\%) and then gradually reduced with increasing age, until they were no longer significant by age 50 . 
Similarly, the relationship between anti-LGB discrimination and recent suicidal behavior was strongest at age 18 and weakened with increasing age, remaining significant until age 26 . These findings highlight emerging adulthood as a period of particular risk for the recent suicidal behavior among LGB individuals.

Cadigan, Lee, and Larimer (this issue) also used a prospective design to examine predictors of mental health service utilization among a community sample of emerging adults including four-year college students, community college students, and nonstudents. Participants were 622 emerging adults (ages 18-23) who completed baseline and 15-month followup assessments to prospectively predict mental health utilization and barriers to service utilization. Mental health service use was reported by $22.7 \%$ of the original sample and $26.1 \%$ identified an unmet need for services. Sex and sexual orientation were predictors of both service use and unmet needs, where women and individuals who identified as a sexual minority were more likely to use mental health services and to identify unmet service use needs. Depression severity (greater severity) and impairment (more impaired) were both associated with a greater likelihood of accessing services and poorer perceived mental health was associated with unmet needs for services. Barriers to service use included that emerging adults felt they could manage on their own, limited time due to other commitments, lack of affordability of services, perceptions that services would not be helpful, and not knowing where to go for services. Nonstudents were more likely not to access services due to lack of affordability or insufficient insurance coverage, whereas students were more likely not to access services because they felt they did not need them.

In the final paper, Villanti et al. (2018) introduce a novel conceptual prevention approach - prevescalation - that targets prevention of escalations in smoking behavior during emerging adulthood. This concept captures the need to address the gap between prevention of smoking initiation, typically implemented during adolescence, and adult-focused tobacco cessation interventions. Because rapid escalation of smoking behavior is likely to occur during emerging adulthood, Villanti et al. (2018) argue that prevescalation strategies, which disrupt transitions between experimentation and established use, are ideally suited for this developmental stage. The authors note that prevescalation strategies may encompass both universal prevention approaches and targeted cessation strategies, and that evaluating prevescalation interventions may require evaluations that go beyond traditional assessment of randomized control trial designs.

Two major themes were identified in this set of studies. First, the studies in this special issue reflect the diversity of emerging adults in the USA. Five of the six empirical studies targeted community samples of emerging adults, including both college-attending youth and those not enrolled in college. Two of the studies in this issue (Cadigan and colleagues, this issue; Fish and colleagues, this issue) point to the particular mental health challenges of sexual minority emerging adults. Hafner and Rushing (this issue) highlight the needs of AI/AN emerging adults and note that their intervention may have had limited effectiveness due to a lack of consultation with emerging adults for the adaptation of their intervention. Findings from these studies highlight the continued disparities that exist among marginalized groups of emerging adults who experience disproportionate rates of mental health, substance use, and other risk behaviors.

The second theme is the articles in this special issue reflect the relatively nascent stage of emerging adult prevention research. For example, the results of the studies that used an RCT design were generally mixed, with some key hypotheses not supported by the data. Such findings may reflect the fact that all of the RCT studies evaluated adaptations of existing evidence-based interventions. A robust literature within the prevention science field indicates the need to strike a balance between fidelity and adaptation, which do not have to be viewed as conflicting goals (Chambers and Norton 2016; Kemp 2016). The studies included in this special issue support the notion that successful adaptation of interventions to suit the unique needs of different developmental stages, such as emerging adulthood, presents some challenges with which the field must reckon. Further, all of these studies in this special issue can be considered examples of type I translation research"bench to bedside" work that encompasses the application of basic science research findings to the development and testing of evidence-based interventions (EBIs).

The concluding commentary by Dr. Laura Hill (Expanding our horizons: Risk, protection, and intervention in emerging adulthood (in this issue)) summarizes the next steps needed to meet these needs and fully advance the field of emerging adult prevention research to the next stage of type II translation, in which proven EBIs are adopted, implemented, and sustained on a large scale (Spoth et al. 2013). The intention of this special issue is to take the first step toward achieving those goals in order to fully realize the potential of preventive interventions to meet the challenges of this transitional life stage.

\section{Compliance with Ethical Standards}

Conflict of Interest The authors declare that they have no conflict of interest.

Publisher's Note Springer Nature remains neutral with regard to jurisdictional claims in published maps and institutional affiliations.

\section{References}

Arnett, J. J. (2000). Emerging adulthood: A theory of development from the late teens through the twenties. American Psychologist, 55, 469. 
Arnett, J. J. (2007). Emerging adulthood: What is it, and what is it good for? Child Development Perspectives, 1, 68-73.

Cadigan, J. M., Lee, C. M., \& Larimer, M. E. (this issue). Young adult mental health: a prospective examination of service utilization, perceived unmet service needs, attitudes, and barriers to service use. Prevention Science. https://doi.org/10.1007/s11121-018-0875-8.

Chambers, D. A., \& Norton, W. E. (2016). The adaptome: Advancing the science of intervention adaptation. American Journal of Preventive Medicine, 51, S124-S131.

Fish, J. N., Rice, C. E., Lanza, S. T., \& Russell, S. R. (this issue). Is young adulthood a critical period for suicidal behavior among sexual minorities? results from a US national sample. Prevention Science. https://doi.org/10.1007/s11121-018-0878-5.

Hafner, S. P., \& Rushing, S. C. (this issue). Sexual health, STI and HIV risk, and risk perceptions among American Indian and Alaska native emerging adults. Prevention Science. https://doi.org/10.1007/ s11121-018-0920-7.

Johnston, L. D. (2010). Monitoring the future: National survey results on drug use, 1975-2008: Volume II: College students and adults ages 19-50. Darby: DIANE Publishing.

Kemp, L. (2016). Adaptation and fidelity: A recipe analogy for achieving both in population scale implementation. Prevention Science, 17, 429-438.

Larimer, M. E., \& Cronce, J. M. (2007). Identification, prevention, and treatment revisited: Individual-focused college drinking prevention strategies 1999-2006. Addictive Behaviors, 32, 2439-2468.

Layland, E. K., Calhoun, B. H., Russell, M. A., \& Maggs, J. L. (2018). Is alcohol and other substance use reduced when college students attend alcohol-free programs? Evidence from a measurement burst design before and after legal drinking age. Prevention Science. https://doi. org/10.1007/s11121-018-0877-6.
Lewis, M., Rhew, I., Fairlie, A., Swanson, A., Anderson, J., \& Kaysen, D. (2018). Evaluating personalized feedback intervention framing with a randomized controlled trial to reduce young adult alcohol-related sexual risk taking. Prevention Science. https://doi.org/10.1007/ s11121-018-0879-4.

Schulenberg, J. E., Sameroff, A. J., \& Cicchetti, D. (2004). The transition to adulthood as a critical juncture in the course of psychopathology and mental health. Development and Psychopathology, 16, 799806.

Schwartz, S. J., \& Petrova, M. (2019) Prevention science in emerging adulthood: a field coming of age. Prevention Science. https://doi. org/10.1007/s11121-019-0975-0.

Spoth, R., Rohrbach, L. A., Greenberg, M., Leaf, P., Brown, C. H., Fagan, A., et al. (2013). Addressing core challenges for the next generation of type 2 translation research and systems: The translation science to population impact (TSci Impact) framework. Prevention Science, 14, 319-351.

Stormshak, E., Caruthers, A., Chronister, K., DeGarmo, D., Stapleton, J., Falkenstein, C., DeVargas, E., \& Nash, W. (2018). Reducing risk behavior with family-centered prevention during the young adult years. Prevention Science. https://doi.org/10.1007/s11121-0180917-2.

U.S. Department of Health and Human Services (HHS), Office of the Surgeon General. (2016). Facing addiction in America: The surgeon general's report on alcohol, drugs, and health. Washington, DC: HHS.

Villanti, A. C., Niaura, R. S., Abrams, D. B., \& Mermelstein, R. (2018). Preventing smoking progression in young adults: The concept of prevescalation. Prevention Science. https://doi.org/10.1007/ s11121-018-0880-y. 